\title{
Prediction and Simulation of electrical and optical characteristics of an OLED based on P3BEdotBT3A organic material
}

\author{
Anass El karkri ${ }^{1, *}$, Imane El mhamedi ${ }^{1}$, and Zakaria $\mathrm{El}$ malki ${ }^{1}$ \\ ${ }^{1}$ Moulay Ismal University. Research team: Modeling, Materials and Systems Control(MMSC). \\ Research laboratory: Computer Engineering and Intelligent Electrical Systems (2ISEI). High School of Technology Meknes \\ (ESTM) BP: 3103, Toulal, Meknes, Morocco
}

\begin{abstract}
The OLED "organic light-emitting diode" has been highly industrialized for several years, especially since their use in smartphones and televisions. OLEDs have attracted a lot of attention because of their desirable characteristics including their low consumption of electrical energy, their flexibility... Our work focuses on the simulation of a single-layer OLED based on an organic material (ITO/P3BEdotBT3A/Ca) using the SILVACO-TCAD simulation software. This simulation allowed us to visualize the operational distribution of Langevin recombination within the proposed structure, and to determine the electrical and optical characteristics, such as luminescence power, current versus electric field, and current versus voltage (the I-V characteristics) for different thicknesses and doping concentration of the emitting layer.
\end{abstract}

\section{Introduction}

After more than twenty years of development, significant progress has been made in the fabrication and design of organic electronic devices. High-performance LEDs, [1-3] solar cells, [4-7] transistors, [8-11] and memory devices [12] have been created and developed.

Organic light-emitting diodes (OLEDs), whose light emission is based on the phenomenon of luminescence, represent light sources capable of providing very high luminance. OLED technology wasn't really tamed until around 2007, the year Sony released an OLED-based television screen. Mastery of this technology has also opened the door to a new market in which companies have not hesitated to enter, despite the lack of profitability, the level of investment required and the challenges that must still be met before this technology equals LED for lighting and LCD for display.

Regarding the structure, OLEDs are composed of an organic semiconductor layer sandwiched between two electrodes (at least one transparent) allowing the application of a DC voltage necessary for their functioning. Currently, the electrodes used are mainly composed of indium tin oxide (ITO) for the transparent anode and an opaque metal (or not) for the cathode.

The evolution of organic semiconductors (which are the basis of organic electronics) was initiated by an important discovery that took place in 1963 when Pope et al demonstrated electroluminescence in single crystals of anthracene [13]. And since that time researchers have started to develop new organic semiconductor materials and improve their properties [14]. They make it possible in particular to synthesize materials with highperformance optoelectronic properties (mechanical characteristics and optical properties) [15-18] for welltargeted applications.

The objective of our work consists on the one hand in determining the electronic and optical properties of an OLED based on the organic material having the structure P3-B-EDOT-B-T3-A [molecule composed of Phenylene (P), Thiophene (T), 3,4-Ethylenedioxythiophene (EDOT) and Benzothiadiazole (B)]. The paper describes the design, characterization and modeling of this OLED using the simulation software TCAD-SILVACO "ATLAS". On the other hand, it shows the effect of varying the thickness of the emitting layer and the doping concentration of charge carriers on these properties.

\section{Computational methodology}

The TCAD (Technology Computer-Aided Design) simulators allow the modeling of the physical and electrical behavior of an electronic component as well as the optical properties, in order to save time and development cost, and also to be able to consider and optimize solutions to improve the performance of devices. SILVACO (silicon Valley Corporation) is a

*Corresponding author: $\underline{\text { a.elkarkri@edu.umi.ac.ma }}$ 
software environment for designing and predicting the performance of semiconductor devices. It is a tool for designing semiconductor devices before they are manufactured. It is very useful in the development of many research projects. The TCAD Silvaco includes new physical models which employ efficient numerical methods and algorithms, new meshing techniques, linear solution optimization, all allowing to obtain simulation results very close to those of the practice. The major advantage of this type of simulator is that it makes it possible to visualize physical phenomena that are difficult to access and therefore to observe. [19]

\section{Electronic properties}

In this work, we will study and simulate the optoelectronic properties of organic light emitting diode based on the P3-B-EDOT-B-T3-A oligomer (figure 1).

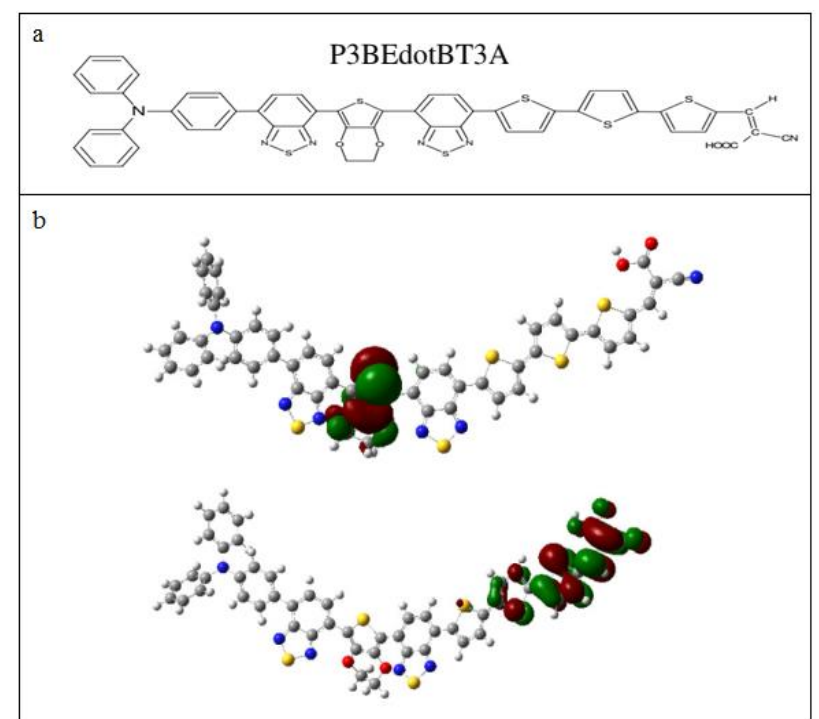

Fig. 1. a/ The sketch map structures of studied organic oligomer. b/ Sketch of B3LYP/6-31G(d,p) calculated energies of the HOMO and LUMO level and the contour plots of HOMO and LUMO orbitals of the studied molecule [18].

The analysis of the HOMO and the LUMO of the oligomer indicates the ability of electron or hole transport [20].

The electronic affinity EA calculated by equation 1 $[21,22]$. It means the capacity of the material to accept one electron from the environment.

$$
E A=\mathrm{E}(\mathrm{M})-\mathrm{E}\left(\mathrm{M}^{-}\right)
$$

Where, $\mathrm{E}(\mathrm{M})$ and $\mathrm{E}\left(\mathrm{M}^{-}\right)$are the total energies of neutral molecule and anion states, respectively. We calculated the energies using the B3LYP/6-31G(d,p) level.

Table 1. The EA and the gap energy Eg of the studied molecule obtained by B3LYP/6-31G(d,p) level. [18]

\begin{tabular}{|c|c|c|}
\hline Molecule & Eg (eV) & EA (eV) \\
\hline P3BEDOTBT3A & 1,96 & 2,23 \\
\hline
\end{tabular}

\section{Physical model}

The fundamental physical equations in semiconductors include the Poisson equation, the two continuity equations for electrons and holes, and the two current equations for electrons and holes. The Poisson equation relates changes in electrostatic potential to local charge densities, the continuity equations describe the time-dependent evolution of the densities of electrons and holes in relation to the mechanisms of transport, generation and recombination, and the transport equations define the current of electrons and holes. Solving these basic equations simultaneously makes it possible to find the electron and hole distributions and the potential. Numerical simulation of a semiconductorbased device is based on solving three fundamental equations that define charge transport in semiconductors: Poisson equation and continuity equations for electrons and holes.

\subsection{Poisson equation}

The Poisson equation is defined as follows [23]:

$$
\operatorname{div}(\varepsilon \nabla \psi)=-\rho_{\mathrm{v}}(\mathbf{r})
$$

Where $\psi$ the electrostatic potential, $\varepsilon$ is is the local permittivity of the medium, $r$ is the position vector and $\rho_{\mathrm{v}}$ is the volume density of charge.

Le champ électrique est déduit du gradient du potentiel électrostatique par

$$
E=-\nabla \psi
$$

\subsection{Continuity equations}

The continuity equations describe the velocity of variation as a function of time of the carrier concentrations. Continuity equations for electrons and holes are given by the following expressions [24]:

$$
\begin{aligned}
& \partial n / \partial t=(1 / \mathrm{q}) \operatorname{div} J_{\mathrm{n}}+\mathrm{G}_{\mathrm{n}}-\mathrm{R}_{\mathrm{n}} \\
& \partial p / \partial t=-(1 / \mathrm{q}) \operatorname{div} J_{\mathrm{p}}+\mathrm{G}_{\mathrm{p}}-\mathrm{R}_{\mathrm{p}}
\end{aligned}
$$

$G_{n}$ and $G_{p}$ are the generation rates for electrons and holes, $R_{n}$ and $R_{p}$ are respectively the recombination rates (internal) for electrons and holes, $\mathrm{J}_{\mathrm{n}}$ and $\mathrm{J}_{\mathrm{p}}$ are the current densities of electrons and holes.

\subsection{Transport equations}

The existence of electric currents in a semiconductor is explained by the presence of an electric field and also by the existence of a concentration gradient of charge carriers in the semiconductor. The currents induced by the electric field are the drift currents and the currents induced by the carrier concentration gradient are called diffusion currents. The diffusion drift model is most commonly used to describe charge transport in a semiconductor. Thus, the current densities $J_{n}$ and $J_{p}$ of the continuity equations can be approximated using Boltzmann's transport theory by the diffusion drift model 
[24]. Using the Fermi quasi-levels of electrons and holes $\left(\Phi_{\mathrm{n}}\right.$ and $\left.\Phi_{\mathrm{p}}\right)$, their expressions are:

$$
\begin{aligned}
& J_{\mathrm{n}}=-\mathrm{q} \mu_{\mathrm{n}} \mathrm{n} \nabla \Phi_{\mathrm{n}} \\
& J_{\mathrm{p}}=-\mathrm{q} \mu_{\mathrm{p}} \mathrm{p} \nabla \Phi_{\mathrm{p}}
\end{aligned}
$$

With $\mathrm{q}$ is the electric charge, $\mu_{\mathrm{n}}$ and $\mu_{\mathrm{p}}$ are the mobility of electrons and holes respectively.

The expressions for the concentrations of electrons and holes are:

$$
\begin{aligned}
& n=n_{i e} \times \exp \left[q\left(\psi-\Phi_{\mathrm{n}}\right) / k T\right] \\
& p=n_{i e} \times \exp \left[q\left(\psi-\Phi_{\mathrm{p}}\right) / k T\right]
\end{aligned}
$$

With $\mathrm{n}_{\mathrm{ie}}$ is the intrinsic effective density.

By introducing these equations into the current density expressions, we obtain:

$$
\begin{aligned}
& J_{\mathrm{n}}=q D_{n} \nabla_{n}-q_{n} \mu_{n} \nabla \psi-n \mu_{n}\left(k T L \nabla\left(\ln n_{i e}\right)\right) \\
& J_{\mathrm{p}}=q D_{p} \nabla_{p}-q_{p} \mu_{p} \nabla \psi-n \mu_{p}\left(k T L \nabla\left(\ln n_{i e}\right)\right)
\end{aligned}
$$

With $D_{n}$ and $D_{p}$ are respectively the Einstein coefficients which have for expression:

$$
\begin{aligned}
& D_{\mathrm{n}}=\mu_{n} \times(\mathrm{kT} / \mathrm{q}) \\
& D_{\mathrm{p}}=\mu_{p} \times(\mathrm{kT} / \mathrm{q})
\end{aligned}
$$

For electric fields greater than $3 \times 10^{5} \mathrm{~V} / \mathrm{cm}$, the current density becomes higher than that predicted in Child's law because mobility increases with the electric field [25] and temperature [26]. In this case the mobility is given by the Poole Frenkel formula [27].

To understand the physical properties of organic devices, it can be taken from the Poole-Frenkel model and the Recombination Langevin model [19].

$$
\begin{aligned}
& \mu_{\mathrm{n}}(\mathrm{E})=\mu_{\mathrm{n} 0} \exp \left[-\mathrm{DELTAEN} . P F M O B / \mathrm{kT} \mathrm{neff}_{\mathrm{n}}+(\text { BETAN. }\right. \\
& \text { PFMOB } / \mathrm{kT}_{\text {neff-GAMMAN.PFMOB })(|\mathrm{E}|)^{1 / 2}}
\end{aligned}
$$

$$
\mu_{\mathrm{p}}(\mathrm{E})=\mu_{\mathrm{p} 0} \exp \left[-\mathrm{DELTAEP} . \text { PFMOB } / \mathrm{k} \mathrm{T}_{\mathrm{neff}}+(\text { BETAP. }\right.
$$$$
\text { PFMOB/kT neff-GAMMAP.PFMOB) } \left.(|\mathrm{E}|)^{1 / 2}\right]
$$

Where,

DELTAEN.PFMOB = Activation energy for electrons, DELTAEP.PFMOB = Activation energy for holes, $\mu_{\mathrm{n} 0}(\mathrm{E})=$ Field dependent mobility, $\mu_{\mathrm{n} 0}=$ Zero field mobility, $\mathrm{E}=$ Electric field, BETAN.PFMOB=Electron Poole-Frenkel factor. BETAP.PFMOB $=$ Hole PooleFrenkel factor. $T_{\text {neff }}$ and $T_{\text {peff }}$ are the effective temperature for electrons and holes respectively.

The recombination is done in two phases. First there is the phase of approximation of the charge carriers then that of formation of the exciton by Langevin capture when the electron / hole distance is less than the critical capture radius $r_{c}$, called the OSANGER distance, and for which the Coulomb interaction energy between the two charges is equal to the thermal energy $\mathrm{k}_{\mathrm{B}} \mathrm{T}$ [28].

$$
r_{\mathrm{c}}=\mathrm{e}^{2} /\left(4 \pi \varepsilon_{\mathrm{r}} \varepsilon_{0} \mathrm{kB}_{\mathrm{B}} \mathrm{T}\right)
$$

$\varepsilon_{\mathrm{r}}$ is the dielectric constant of the organic material, $\varepsilon_{0}$ is the dielectric permittivity of vacuum, and $\mathrm{k}_{\mathrm{B}}$ is the Boltzmann constant.

The Langevin recombination rate coefficient is given by [29, 31]:

$$
R_{\mathrm{L}}(\mathrm{x}, \mathrm{y}, \mathrm{t})=\mathrm{A} \text { LANGEVIN }\left(\mathrm{q}\left[\mu_{n}(E)+\mu_{p}(E)\right] / \varepsilon_{\mathrm{r}} \varepsilon_{0}\right)
$$

Where, $\mathrm{R}$ represents the rate of recombination.

The Langevin recombination rate coefficient expressed as:

$$
R_{\mathrm{Lnp}}=r_{\mathrm{L}}(\mathrm{x}, \mathrm{y}, \mathrm{t})\left(\mathrm{np}-\mathrm{ni}^{2}\right)
$$

where $\mathrm{ni}$ is intrinsic carrier concentration, $\mathrm{r}_{\mathrm{L}}(\mathrm{x}, \mathrm{y}, \mathrm{t})$ is the Langevin recombination rate coefficient.

\section{Presentation of the simulated structure}

In this work, the simulated structure is an organic LED composed of a single layer which is shown in (Figure 2), the device configuration is: ITO / P3BEdotBT3A / Calcium $(\mathrm{Ca})$.

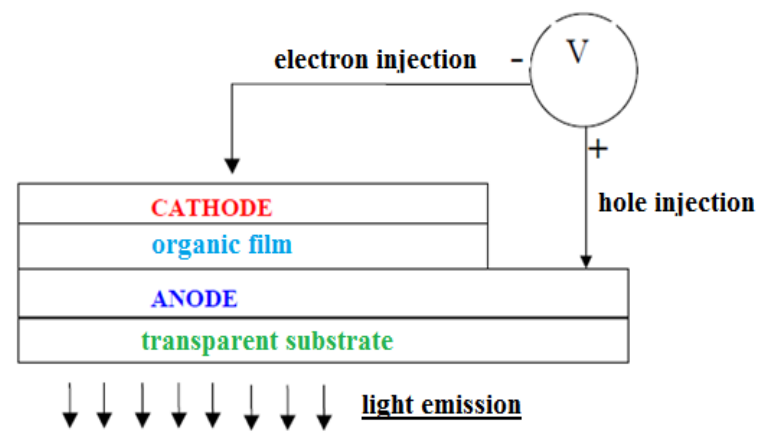

Fig. 2. Structure of an organic light-emitting diode.

Organic electroluminescence is based on the injection of charge carriers into the material. The organic lightemitting diodes thus have a multilayer structure with two electrodes which sandwich a film of organic electroluminescent material according to the scheme of (Figure 2) [32].

The anode is made from a layer of indium tin oxide (ITO) deposited on a glass substrate. It is a conductive transparent oxide, commonly used in various optoelectronic devices as a transparent electrode [24], because of its excellent optical and electrical properties (protection against electromagnetic fields, thermal insulation...).

The cathode is usually obtained from the evaporation of a layer of calcium. The low work function of calcium $(2.9 \mathrm{eV})$ improves the injection of electrons into organic layers [33].

The organic material has an energy band structure such that there is a gap between the HOMO (Highest Occupied Molecular Orbital) orbitals which act as a valence band and the LUMO (Lowest Unoccupied Molecular Orbital) orbitals which play the role of conduction band (Figure 1). The sandwich structure (Figure 2) used in the achievement of organic light- 
emitting diodes allows the injection of electrons through the cathode into the conduction band and the injection of holes into the valence band through the anode. Under the effect of the applied field, the carriers will migrate and recombine to form an exciton, the latter will then disappear radiatively to give rise to the phenomenon of electroluminescence [32].

\section{Results and discussion}

\subsection{Effect of thickness}

In this part we study the effect of the variation in the thickness of the active layer (P3BEdotBT3A) on the eclectic and optical characteristics of the device, while keeping the doping concentration fixed at $10^{15} \mathrm{~cm}^{-3}$.

Figure 3 shows a high rate of Langevin recombination over the entire surface of the active layer and especially on the side of the cathode, with a slight decrease when the thickness is increased. This means obtaining good characteristics of the OLED.

Figures 4, 5, and 6 show the I-V characteristics, the luminescence as a function of the applied voltage, and the current as a function of the electric field, respectively, for different thicknesses of the active layer (40, 50, 60, and $70 \mathrm{~nm})$.

From the results, the potential luminescent power increases as the anode voltage increases, and the light intensity decreases as the thickness of the emitting layer (P3BEdotBT3A) increases, so it can be concluded that it is better to make the emitting layer thinner, because increasing the thickness of the active layer increases the direct charge trapping and reduce the current of the OLED.

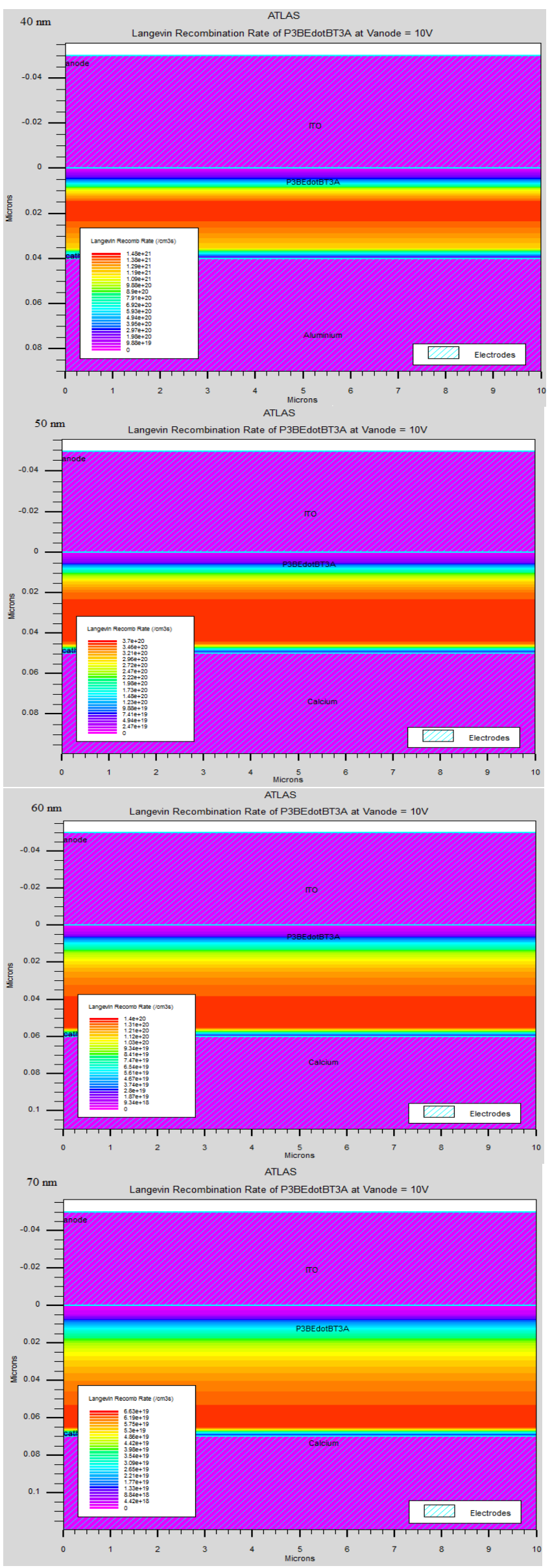

Fig.3. Langevin recombination rate for different thicknesses of the active layer. 


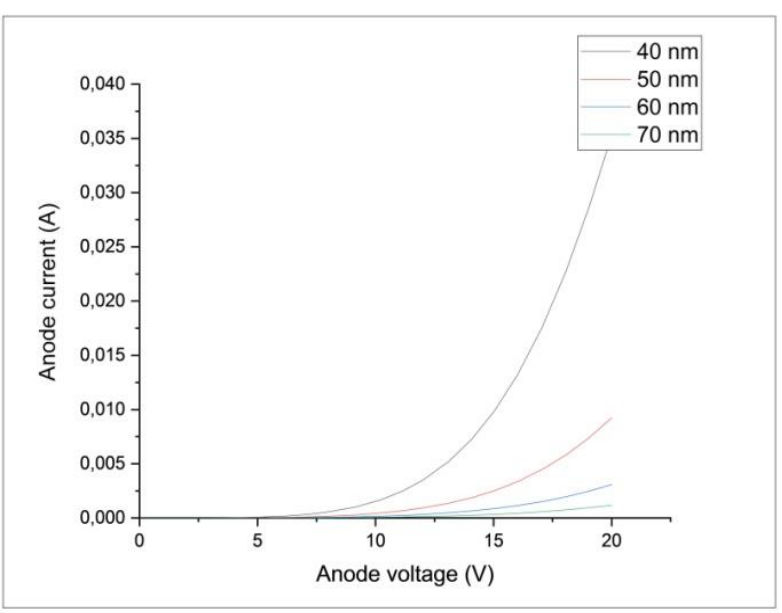

Fig.4. I-V Characteristics of OLED for different thickness of emitting layer.

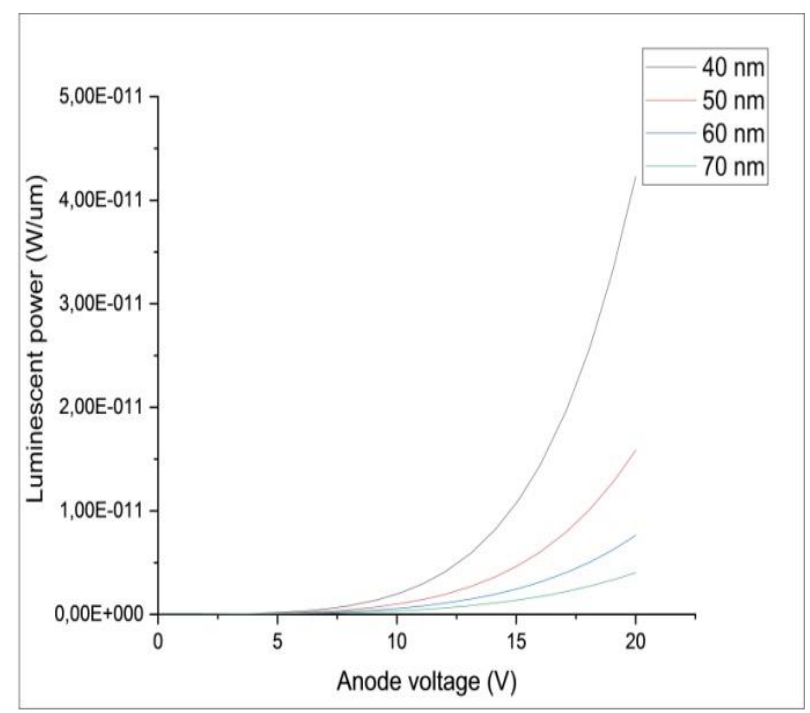

Fig.5. Luminescent power vs anode voltage for different thickness of emitting layer.

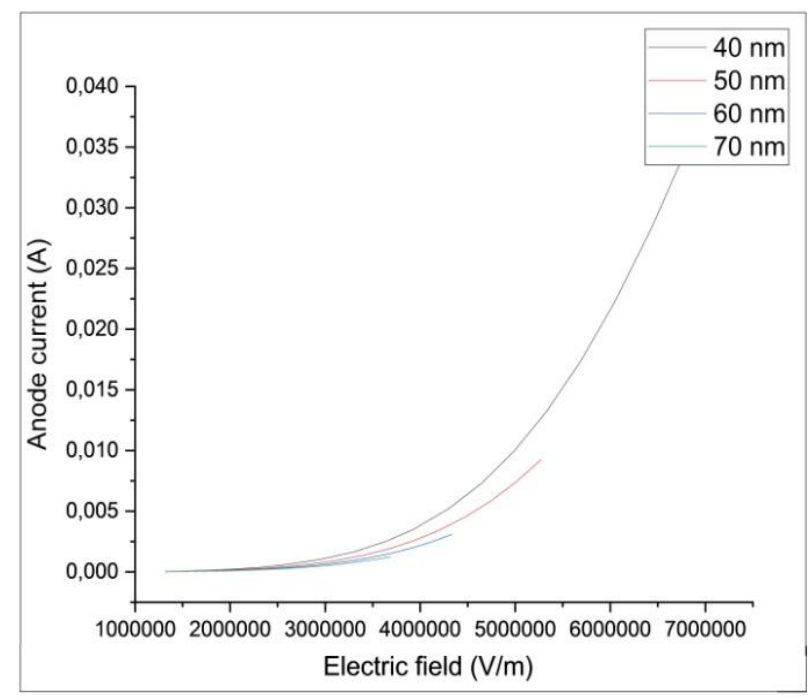

Fig.6. Anode current vs Electric field for different thickness of the active layer

\subsection{Effect of doping concentration}

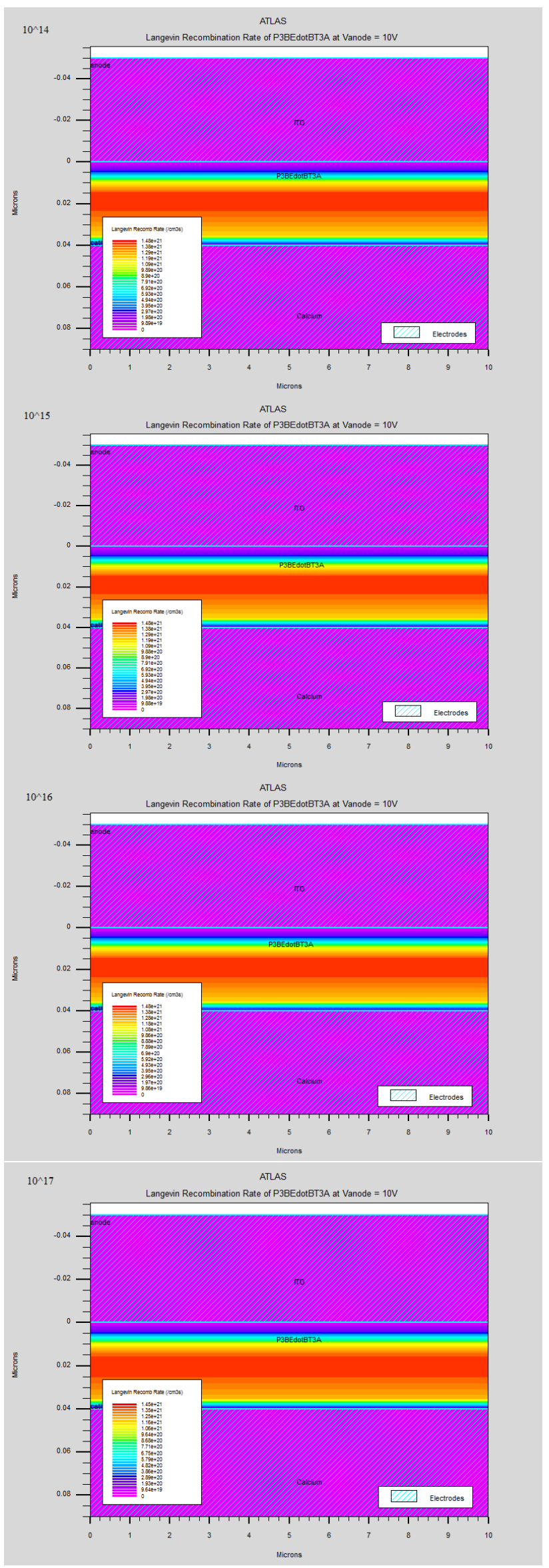

Fig.7. Langevin recombination rate for different doping concentration of the active layer 
In this part of the work, we will study the influence of the variation in doping concentration on the characteristics of OLED based on (P3BEdotBT3A) molecule. The results obtained (Figures 7, 8, 9, and 10) show only a slight variation in the physical quantities characterizing the OLED, when this concentration is varied, because the performance of the device does not improve significantly from a doping rate above $1015 \mathrm{~cm}-$ 3 , due to the triplet extinction mechanisms, such as triplet-triplet annihilation, triplet-polaron annihilation, and electric field oriented dissociation of excitons. [34, 35]

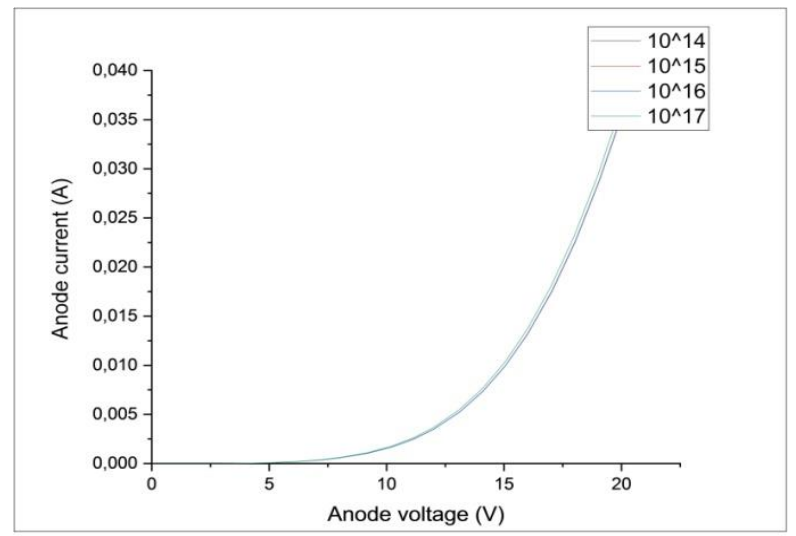

Fig. 8. I-V Characteristics of OLED for different doping concentration of the active layer.

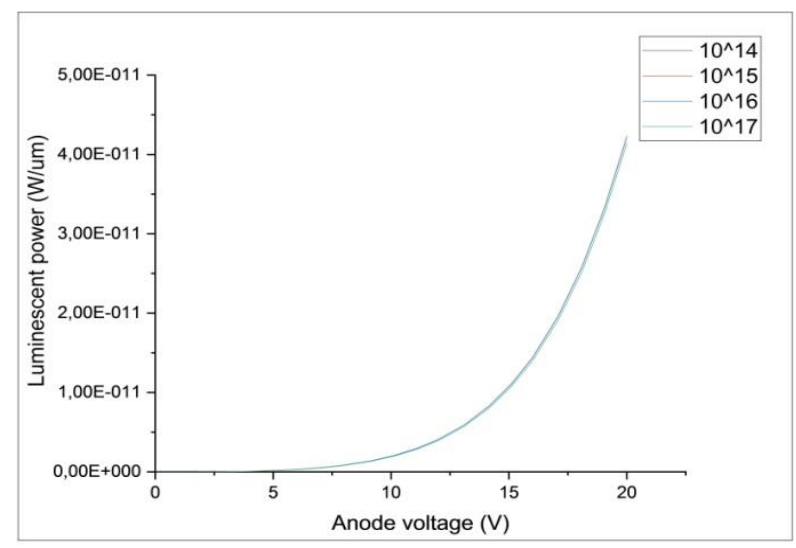

Fig. 9. Luminescent power vs anode voltage for different doping concentration of the active layer

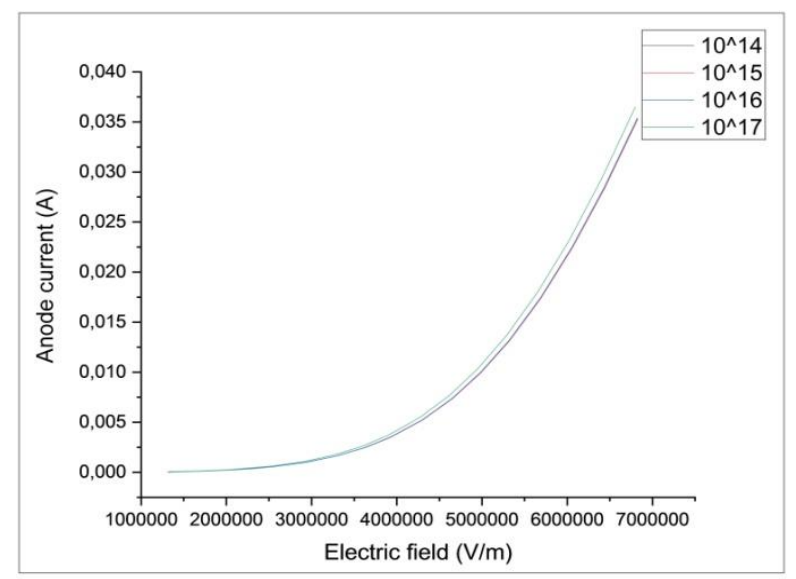

Fig.10. Anode current vs Electric field for different doping concentration of the active layer

\section{Conclusion}

In this paper, we predicted the electrical and optical characteristics of an OLED based on an organic material (P3BEdotBT3A) as an emitting layer, using Silvaco TCAD software. We presented the electronic properties of the active layer, the physical model and the structure of the studied monolayer OLEDs. The results are obtained by solving differential equations describing the mechanism of transport and recombination of polymers and calculating the rate of radiation for luminescence due to Langevin recombination in OLED. We obtained the I-V characteristics, the luminescence power characteristics, and the current as a function of the electric field, for different thickness and doping concentration of the emitting layer. It is concluded that the reduction in thickness results in an improvement in the characteristics of the device regardless of the doping concentration which does not have a great influence when using the P3BEdotBT3A molecule.

The simulation performed allowed us to look at and analyze the physical processes inside the structure, optimize the diode design and avoid time-consuming and expensive experiments on real devices.

This work has been supported by the Volubilis project AI n: MA/11/248 and by CNRST/CNRS cooperation (Project chimie 1009). We are grateful to the Association Marocaine des Chimistes Theoriciens (AMCT) for its valuable help concerning the programs.

\section{References}

1. S.-J. Su, E. Gonmori, H. Sasabe, J. Kido, Adv. Mater. 2009, 21, 361. (2009)

2. S.-J. Su, T. Chiba, T. Takeda, J. Kido, Adv. Mater. 2008, 20, 1. (2008)

3. F. Huang, P.-I. Shih, C.-F. Shu, Y. Chi, A. K.-Y. Jen, Adv. Mater. 2009, 21, 361. (2009)

4. E. Wang, L. Wang, L. Lan, C. Luo, W. Zhuang, J. Peng, Y. Cao, Appl. Phys. Lett, 2008, 92, 033307. (2008)

5. M. D. Irwin, D. B. Buchholz, A. W. Hains, R. P. H. Chang, T. J. Marks, Proc. Natl. Acad. Sci. USA, 2008, 105, 2783. (2008)

6. J. Hou, H.-Y. Chen, S. Zhang, G. Li, Y. Yang, J. Am. Chem. Soc. 2008, 130, 16144. (2008)

7. J. Peet, J. Y. Kim, N. E. Coates, W. L. Ma, D. Moses, A. J. Heeger, G. C. Bazan, Nat. Mater. 2007, 6, 497. (2007)

8. H. Yan, Z. Chen, Y. Zheng, C. Newman, J. R. Quinn, F. Do“tz, M. Kastler, A. Facchetti, Nature, 2009, 457, 679. (2009)

9. M. Tang, A. Reichardt, N. Miyaki, R. M. Stoltenberg, Z. Bao, J. Am. Chem. Soc. 2008, 130, 6064. (2008)

10. H. Klauk, U. Zschieschang, J. Pflaum, M. Halik, Nature. 2007, 445, 745. (2007) 
11. H. N. Tsao, D. Cho, J. W. Andreasen, A. Rouhanipour, D. W. Breiby, W. Pisula, K. Mu"llen, Adv. Mater. 21, 209, (2009).

12. J. C. Scott, L. D. Bozano, Adv. Mater. 19, 1452. (2007)

13. M.HISSLER «Les diodes électroluminescentes organiques : technologies et performances », Campus de Beaulieu - University of Rennes 1. (2013)

14. H.CHOUKRI - doctoral thesis «Contrôle de la couleur d'émission d'une Diode Electroluminescente Organique (OLED) multicouche via la diffusion des excitons $»$, University of Paris-Nord -Paris XIII, (2008)

15. Z. EL Malki, M. Bouachrine, F. Serein-Spirau, JM. Sotiropoulos, International Journals of Advanced Research in Computer Science and Software Engineering ISSN: 2277-128X (Volume- 8, Issue12), 38-51 (2018)

16. Z. EL Malki, M. Bouachrine, M. Hamidi, F. SereinSpirau, J. P. LerePorte, J.M. Sotiropoulos, J. Mater. Environ. Sci. 7 (9) 3244-3255 (2016)

17. Z. EL Malki, M. Bouachrine, L. Bejjit, M. Haddad, F. Serein-Spirau, J-M. Sotiropoulos, International Journals of Advanced Research in Computer Science and Software Engineering ISSN: $2277-$ 128X (Volume-7, Is sue-6), 96-107 (2017)

18. Z. El Malki, A. El karkri, M. Bouachrine,F.S. Spirau3. Springer Nature Switzerland AG 2020, https://doi.org/10.1007/978-3-030-36475-5_28

19. ATLAS Users Manual device simulation software. SILVACO International, Santa Clara, (2004).

20. A. De Oliveira Marcos, A. Duarte Hélio, J.M. Pernaut, B. De Almeida Wagner, J. Phys. Chem. 104, Issue 35, 8256. [CrossRef] https://doi.org/10.1021/jp001252p (2000)

21. P. Politzer, F. Abu-Awwad, Theor. Chem. Acc. 1998, 99, 83. [Cross Ref] https://doi.org/10.1007/s002140050307

22. M. Anafcheh, R. Ghafouri, N.L. Hadipour, Sol. Energy Mater. Sol. Cells. (2012), 105, 125. [CrossRef] https://doi.org/10.1016/j.solmat.2012.05.015

23. C. Haiying, C. Junwu, Q. Chengfeng, B. Z. Tang, Man Wong and Hoi-Sing Kwok, "Blue organic light-emitting diode based on 1,2,3,4,5-pentaphenyl1-(8-phenyl-1,7-octadiynyl)silole," in IEEE Journal of Selected Topics in Quantum Electronics, vol. 10, no. 1, pp. 10-15, Jan.-Feb. (2004).

24. Md.R.SERDOUK, magister thesis « Etude par simulation numérique des propriétés électriques d'une cellule solaire en silicium amorphe hydrogéné (a-Si:H) », University of Mohamed Khider - Biskra, (2015)

25. D.M. Pai, B.E. Spingett, Rev. Mod. Phys.65, 163, (1993)
26. P.M. Borsenberger, L. Pautmeier, H. Bassler, "Charge Transport in Disordered Molecular Solids" J. Chem. Phys. 94, p. 5447, (1991)

27. D.M. Pai. J. Chem. Phys. 52, p. 2285, (1970)

28. J. Kalinowski, "Organic light-emitting diodes: Principles, characteristics, and processes", New York: Marcel Dekker, (2005)

29. C.H. Cheung, \& Ng, A.M. Ching \& Djurišić, A.B. \& Liu, Z.T. \& Kwong, C.Y. \& Chui, P \& Tam, HoiLam \& Cheah, K.W. \& Chan, Wai \& Chan, J \& Lu, A.W.\& Rakić, A.D.. "Angular dependence of the emission from low Q-factor organic microcavity light emitting diodes". Displays. 29. 358-364. (2008)

30. G. R. Chaji and A. Nathan, "Parallel Addressing Scheme for Voltage-Programmed Active-Matrix OLED Displays," in IEEE Transactions on Electron Devices, vol. 54, no. 5, pp. 1095-1100, May (2007)

31. Blom, PWM, MJM. de Jong, and S. Breedijk, "Temperature Dependent Electron-Hole Recombination in Polymer LightEmitting Diodes", Applied Physics Letters; 71(7): 930-932. (1997)

32. S.PANOZZO, doctoral thesis «Etude des propriétés électroniques de nouveaux composés organiques électroluminescents », University of Joseph-Fourier de Grenoble I, (2003)

33. C.PINOT - doctoral thesis « Modélisation des diodes électroluminescentes organiques multicouches dopées. Application a de nouvelles architectures », École Polytechnique, France (2008)

34. S. H. Rhee, K. S. Yook, S. H. Kim, and S. Y. Ryu, ECS J. Solid State Sci.Technol., 5, R1 (2016)

35. S. H. Rhee, C. S. Kim, M. K. Song, K. B. Chung, and S. Y. Ryu, ECS Solid State Lett., 3, R49 (2014) 